\title{
Modifying and co-creating the urban soundscape through digital technologies
}

\author{
Dick Botteldooren [0000-0002-7756-7238], Ghent University, Research group WAVES. \\ Ghent, Belgium. Dick.Botteldooren@ugent.be
}

Toon De Pessemier [0000-0002-3920-7346], Ghent University, WAVES, Belgium. toon.depessemier@ugent.be

Karlo Filipan [0000-0002-2966-5984], Ghent University, WAVES, Belgium. karlo.flipan@asasense.com

Kang Sun [0000-0002-0338-0104], Ghent University, WAVES, Belgium. kang.sun@ugent.be

Bert De Coensel [0000-0003-18/5-6436], Ghent University, WAVES, Belgium. bert.decoensel@ugent.be

Timothy Van Renterghem [0000-0003-0435-5485], Ghent University, WAVES, Belgium. timothy.vanrenterghem@ugent.be

\begin{abstract}
Sound is one of the most dynamic elements of the public open space in cities. The perception and understanding of this sonic environment by its users or society as a whole is commonly referred to as the soundscape. It depends on the noticeability of the composing sounds; the preference, expectations and beliefs of the users; and the overall context that is set by the visual environment and the envisaged use of the space. The local character and the volatility of the soundscape make it an ideal subject for co-creation involving citizens. Digital technologies are applicable for audiovisually predicting the impact of design options. Auralisation, either ab initio or based on multichannel recordings, still involves technological challenges that will be explored in this chapter. Digital technologies can also be used for adding sound accents that allow to change the character of the soundscape, e.g. making it livelier or increasing its mental restoration potential. Such digitally augmented soundscapes can be the direct result of a co-creation effort with the users of the public open space. The innovative combination of creating a tailored soundscape and the ability to achieve this through a co-creation process has a promising potential impact on the user experience in public open spaces.
\end{abstract}

Keywords - Soundscape, co-creation, virtual reality, augmented space

(C) The Author(s), 2020

Smaniotto Costa, C. et al. (Eds.): C3Places, Culture \& Territory 04, pp. I85-200, 2020

https://doi.org//0.24I40/2020-sct-vol.4-2.2 


\section{AN INTRODUCTION TO URBAN SOUNDSCAPE}

The urban public space contributes to the health and wellbeing of the inhabitants of growing and more densely populated urban areas (Björk et al. 2008). The urban public space is perceived and understood by its users through a combination of senses, yet while designing this space, visual aspects often remain the main and only point of concern. Sound, smell, micro-climate, etc. are more volatile components of the urban space that are strongly related to the use of the city. Yet they contribute to an important extent to the overall liveliness, pleasantness, and restorative character of the public space. They make it a place suitable for a specific use that contributes to the overall identity of the city (Rehan, 2016). This chapter focuses on the sound environment.

\section{Perception of the urban sound environment}

The sound environment as perceived and understood by the users of the public space within context - for which the term urban soundscape is now commonly used - is more relevant for urban public space design than the mere sound levels per se (Raimbault \& Dubois, 2005). The main determinants of soundscape and its effects on urban dwellers are now well known (Kang et al., 2016). Sound perception is a process that could be described as the whole auditory scene analysis and its concurrent interpretation by the person. Environmental sound is a complex mixture of various sounds originating from different sources. In order to understand it, humans tend to dissolve this mixture into the individual auditory streams using auditory, but also visual as well as other cues (Bregman, 1994).

Environmental sounds can be regarded as any sound that does not have a communication value for the listener, as opposed to speech or other informational sounds. Therefore, initially, there is no particular strong attention focus on the environmental sound, and the person is listening in readiness. Consequently, most of the environmental sounds that humans are exposed to are therefore the ones that are not being regularly noticed but form a background mix or a hum. However, from these sounds the listener's attention selects and forms the auditory streams. Auditory attention is, at one hand, guided by the physical characteristics of the sound that contribute to its saliency, i.e. standing out of its background (Filipan et al., 2019). On the other hand, people also assign meaning to the sound and focus their attention based on their preferences for listening (Filipan et al., 2017).

Sounds that have positive connotation and consequential attention to them is something that would be considered as an improvement of the sonic environment. Accordingly, in the soundscape research, it was shown that by adding positively contextualized sounds, such as bird sound or the sound of water streams, the characteristics of the added sounds improve the overall appreciation of the sonic environment (De Coensel et al., 20I I). 


\section{Categorization of urban soundscapes}

From an urban planning point of view, the vision on the use of specific public spaces in the urban network determines the desired matching soundscape. For this, a categorization of soundscapes and classification of sounds (Brown et al., 20I I) could be useful. In recent years, categorization of soundscape has often been related to the circumplex model of affect (Axelsson et al., 2010). This model distinguishes between several areas in a two-dimensional pleasantness-arousal plane. In between the main axes which are labelled pleasant-unpleasant and eventful-uneventful respectively interesting quadrants of soundscapes are identified that are labelled exciting, chaotic, monotonous, and calm. These areas could also be linked to the sounds that are dominantly heard in the public place. For example, the sound of people is often dominant in soundscapes that are labelled exciting, mechanical sounds are prominent in soundscapes that are labelled chaotic, and natural sounds are often dominant in soundscapes that are labelled calm. This approach and all experimental work using it assumes attentive listening.

However, as discussed above, the users do not commonly notice the sounds while using the public space. Hence a very common category of urban sound environments is simply backgrounded. These sounds are not expected to significantly contribute to the experience of the place. Thus, another categorization that focusses more strongly on the role of the soundscape in the overall perception of the urban public place was proposed (Fig. I). This hierarchical categorization distinguishes between backgrounded and foregrounded, a property that is strongly related to the

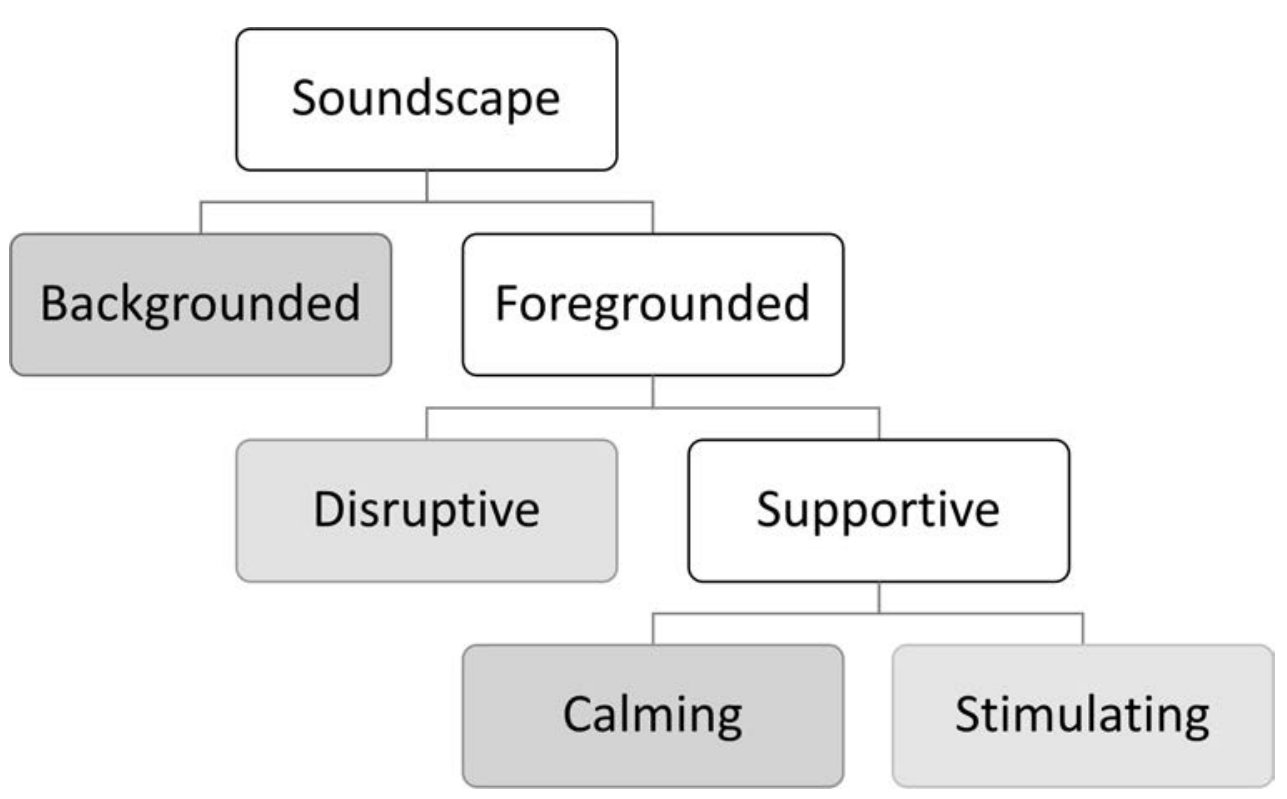

Fig. I: Categorization of urban soundscapes according to their contribution to the experience of the urban public place. Source: Authors. 
degree to which the soundscape attracts attention, for example due to the salient components. Foregrounded soundscapes that prohibit the intended use of the place or at least disrupt it, are labelled disruptive while all others are rather supportive. Finally, supportive soundscapes can support the calming or the stimulating character of the public place. This last subdivision is clearly related to the exciting and calming areas in the affect model.

\section{Opportunities for co-creation}

Urban sound is often a by-product of activities: driving cars or public transport, cooling and heating of houses, enjoying pub-life, etc. As these sounds are often unwanted, several technologies and planning (Sanchez et al., 2018) could be applied to mitigate them. However, all of these come at an economic or social cost. As the use of the place, the context, and expectations of its users play an important role in how the sound environment is perceived and understood, the local inhabitants should typically be involved in deciding what measures are appropriate and require priority (Schulte-Fortkamp \& Jordan, 2016). Co-creation therefore opens a wealth of opportunities to improve public spaces and their use. This co-creation should nevertheless avoid a few critical pitfalls. Firstly, when it comes to the technical side of mitigation lay people often lack the technical expertise to estimate the expected impact of noise control. For example, the difference in effectiveness of a green berm and a noise wall may be difficult to estimate (Van Renterghem and Botteldooren, 2012), or the effect of traffic signal synchronization on noise and air pollutant emission may be confused (De Coensel et al., 2012). Therefore, a training session for the people involved in co-creation may be needed (Botteldooren et al., 2018). In addition, urban sound experts predicting the impact of noise mitigation use indicators such as a yearly-averaged equivalent noise level, LAeq, that are difficult to interpret by the stakeholders participating in the co-creation process.

In recent years virtual and augmented reality (AR/VR) (Calabrese \& Baresi, 2017; Fukuda et al., 2017) have been introduced to preview urban design and architecture both off site and on site. Unfortunately, the sonic environment is often not, or only with very poor ecological validity, included in the AR/VR environment. This technology nevertheless opens a unique opportunity to increase the level of understanding of urban sound design by the stakeholders in the co-creation process. This chapter will elaborate on these opportunities and remaining challenges.

But technology can lead to another, less expected, co-creation. Musicians have since the realm of cities collaborated in co-creating the soundscape of public places. Today, technology can extend this possibility with new ways of delivering sounds that augment the urban soundscape and by largely extending the range of sounds that can be used. In a passive way, loudspeakers providing sounds that are generally liked by users of the space, are added to benches (Schulte-Fortkamp \& Jordan, 20I6) or carefully integrated in the landscape (Licitra et al., 2010). Users of the public place 
are usually participating in the design process of the sound compositions. But co-creation could go one step further allowing users of the space to augment the space with their own designs. Musikiosk (Steele et al., should be 2019 (instead of 20I5)) simply provided the technology for people to share sounds and music brought on their portable devices. More opportunities for instantaneously co-creating the environment could nevertheless be envisaged.

\section{THE USE OF VIRTUAL REALITY IN CO-CREATING SOUNDSCAPES}

A co-creation process requires a good representation of the object of design that is understandable by all stakeholders. The perception of the sonic environment requires full embedding and a realistic context. Hence, the spatial nature of a sonic environment as well as the visual context need exceptional care. Through three examples where virtual reality was recently applied in urban soundscape composition, the available technology and its use are discussed.

\section{Evaluating the urban soundscape in a virtual context}

Co-creation requires stakeholders to be aware of the issue at stake. Lay people are often not aware of the influence that sound has on the perception of the urban environment. When a public place appears in popular media, the image may be rather correct, but the natural sound is often replaced by or mixed with music and narratives. Thus, some education of the people is required. To this end, a database of recordings of urban public places in large cities across the globe was constructed. The selection of public places was guided by local inhabitants. For this, using an online interface (Fig. 2) they located places in the city with a distinct soundscape: chaotic and restless, full of life and exciting, lifeless and boring, calm and tranquil.

Considering public open spaces (squares, parks, traffic-free streets etc.) within the city center of Montreal...

...where do you experience the soundscape to be the most full of life and exciting during the day? Please drag the marker on the map to that location (zoom in/out if needed).

You can (optionally) clarify your choice below, indicate the typical time and day of the week, etc:

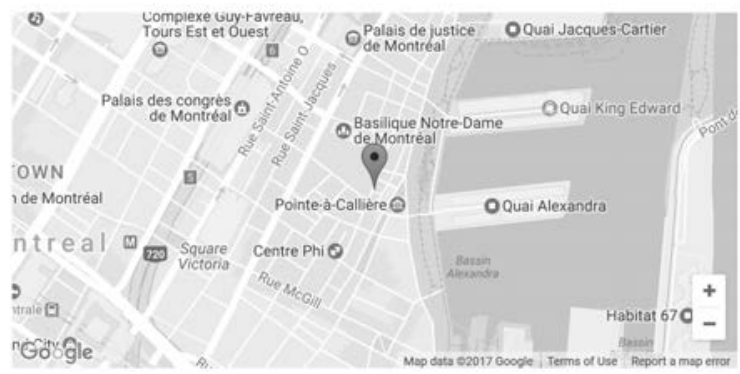

Fig. 2: Web interface used by local inhabitants for selecting public places where typical soundscapes can be found. Source: Authors.

At the locations most frequently selected by the local inhabitants, recordings of 360degree video (GoPro Omni spherical camera system, consisting of 6 synchronized GoPro HERO 4 Black cameras) and first-order ambisonics (Core Sound TetraMic microphone with windshield and Tascam DR-680 Mkll 4-channel recording device) 
were made during 10 to 15 minutes. For reference, binaural audio (HEAD acoustics HSU III.2 artificial head with windshield and SQobold 2-channel recording device) was also added. Fig. 3 shows the recording equipment on a square in Boston, USA.
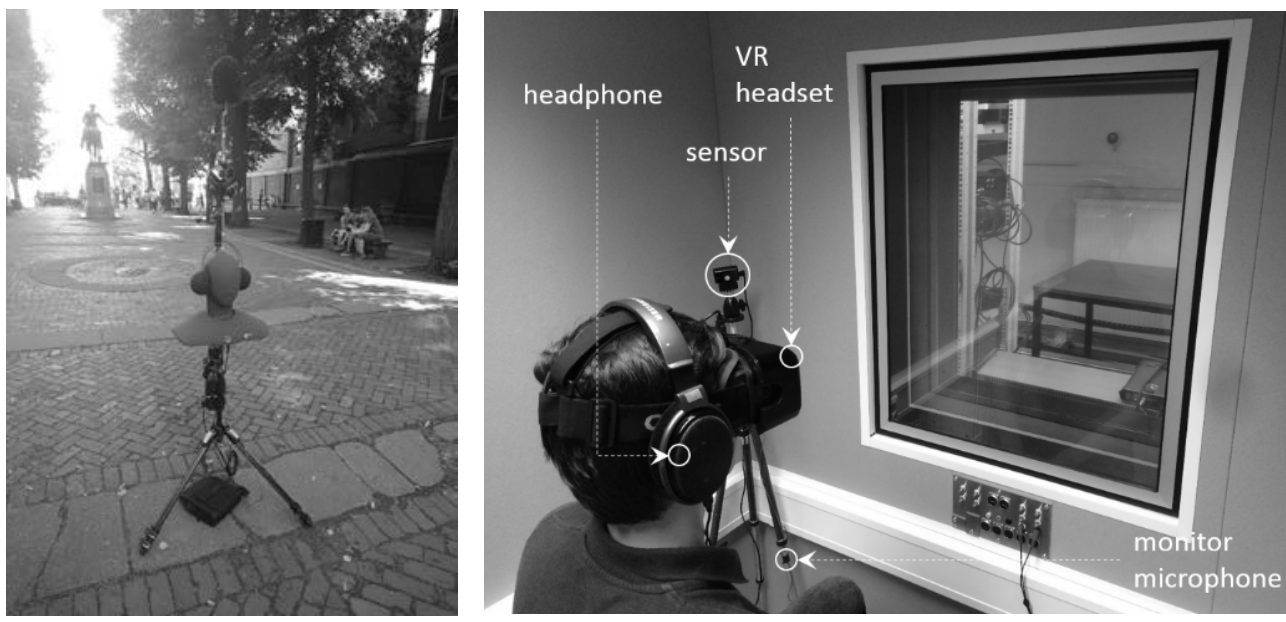

Fig. 3: Left: Recording setup used to collect 360-degree video, ambisonics and binaural sound field; Right: Playback setup with VR headset and headphones. Source: Authors.

Post-processing of audio and video was performed using a range of software, including Kolor Autopano Video and Autopano Giga for stitching and time synchronization of video from 6 separate cameras into one single 360 degree video file, and visually masking of tripod and binaural/ambisonics microphones in the video; HEAD acoustics ArtemiS 8.3 for processing of binaural recordings and calculation of acoustical properties; VVMic 3.5 for processing of ambisonics recordings, conversion from A-format to B-format using microphone-specific calibration/equalization files; FFmpeg for synchronization of audio and video, colour calibration of video, and final selection of segments and combination of media into .mov container; and Google Spatial Media Metadata Injector for adding 360-degree video and spatial audio metadata to the videos.

The visual presentation is achieved by GoPro VR Player (version 3.0) software, which allows to play back video including spatial audio. The 360-degree video is presented through an Oculus Rift head-mounted display, and the participant can freely move its head and look around in all directions. The audio is played back through Sennheiser HD 650 headphones, driven by a HEAD acoustics LabP2 calibrated headphone amplifier. In Sun et al. (2018a) realism and immersion of ambisonics and binaural reproduction have been evaluated with a test panel. No significant differences were shown between ambisonics and binaural reproduction on the perceptual dimensions: envelopment, immersion, representation, readability, realism, and overall quality provided that the head was fixed in the direction corresponding to the binaural recording. On the above dimensions a score of 4 out of 5 was typically reached. 
Currently, the database contains around 100 urban recordings collected in 9 cities'. For these recordings to be useful as examples of good and bad practice, they need to be classified and indexed. The most abstract level of classification could follow the scheme of Fig. I. To that end, panels of 20 persons (age between 25 and 35 years, gender balanced) were invited to experience the recorded environments in virtual reality (Oculus Rift head-mounted display, Sennheiser HD 650 headphones, driven by a HEAD acoustics LabP2 calibrated headphone amplifier). Participants experienced the VR setting during one minute after which they answered a set of questions related to the contribution of the sonic environment to their overall experience. More details of this experiment can be found in Sun et al. (2019). As classification by listening panels is very labour intensive, models were constructed that predict the degree of belonging to any of the soundscape classes based on (visual and) acoustic quantities.

In Fig. 4 each of the recordings is represented by a dot in the two-dimensional plane spanned by the first principle components of the classification of foregrounded soundscapes. Backgrounded soundscapes classify in an orthogonal dimension, yet their projection is also shown. Prototypical examples can be retrieved by selecting specific dots in this classification scheme.

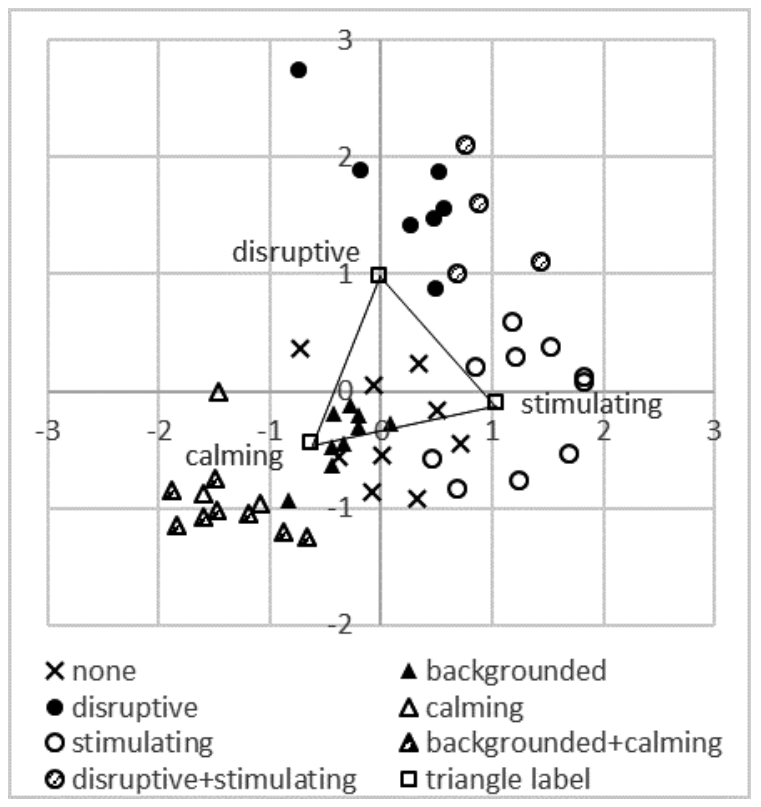

Fig. 4: Fuzzy classification of soundscapes shown in the plane of the first principle components. Source: Authors. 


\section{Intervention on a bridge}

Co-creation of the design of the public place could benefit from the ability of lay persons and stakeholders in general to evaluate alternative scenarios in virtual reality. In this example, it is shown how distinctive designs of a bridge over a ring road including noise barriers and focusing on matching design styles, could be compared and evaluated by stakeholders. The ecologically valid way to experience a bridge is while walking across it. Thus, also in virtual reality, this walking experience should be simulated. For this, the broader context was recreated in 3D Studio Max software and Unity Game Engine. For reproducing the sonic environment, one could also follow the ab initio approach and combine the sounds of individual cars, trucks, talking people, etc. available for the Unity engine. Although this approach, referred to as auralisation (Georgiou \& Hornikx, 20I7), has made tremendous progress over the last years, it remains difficult to create the correct sonic ambience that allows people to identify their familiar city. Hence, a hybrid approach was used. The general sonic environment was recorded using four channel ambisonics (Soundfield ST350 Portable Microphone System), while the sound of specific salient elements in the visual scene such as a tram or a person walking, was added using Unity. To account for the presence of a new noise barrier, the recorded sound was attenuated using a spectral filter matching the numerically simulated physical attenuation (Sanchez et al., 2017).

Four alternative designs (Fig. 5) were created and presented to a panel of 75 individuals. Each visual design was accompanied by the matching sonic design. Sonic environments differed in the contribution of the highway which corresponded to

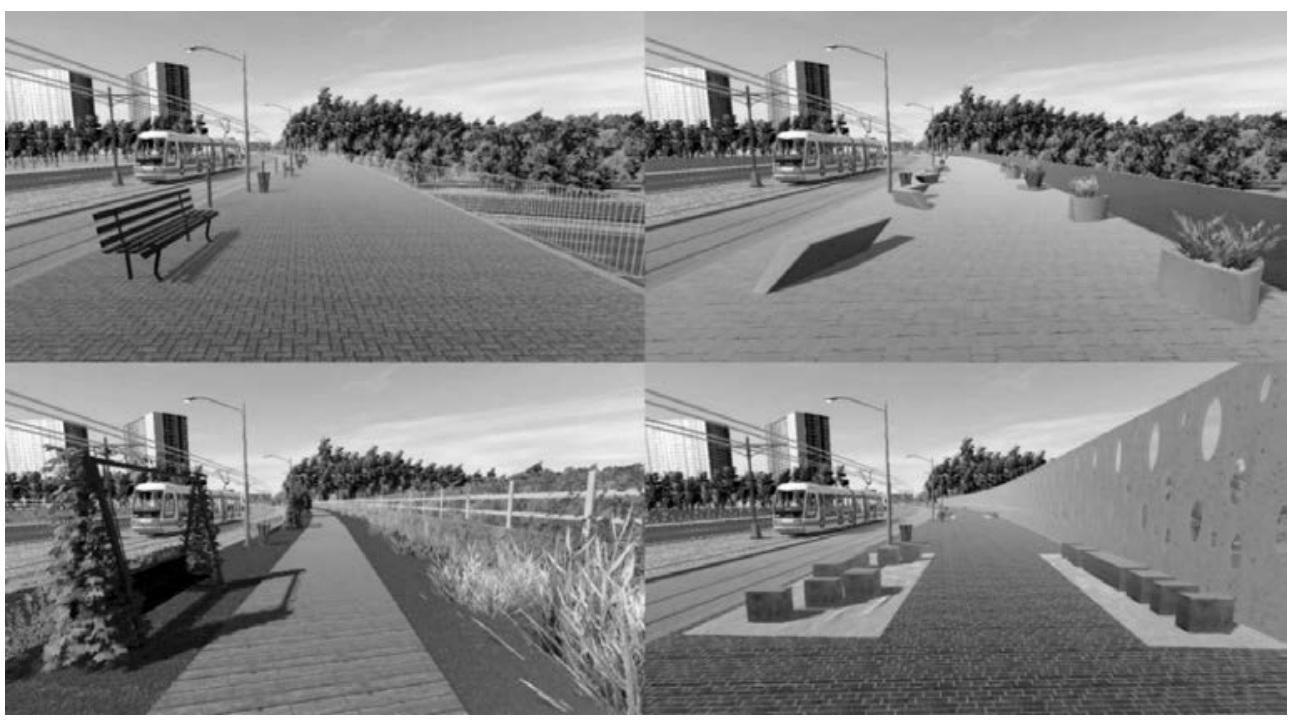

Fig. 5: Four design alternatives presented in the virtual environment to the lay people. These designs are labeled traditional, modern, vegetated, and whimsical from upper-left to bottom-right. Source: Authors. 
average sound levels of $76.5,68.6,65.3,64.1 \mathrm{~dB}(\mathrm{~A})$, respectively depending on the screen height bordering the bridge. The sound of the tram, other persons walking in the scene, and one's own footsteps were kept the same in the four scenarios. The latter is important as it sets the frame of reference for the listener (Aletta et al., 20I6). As the purpose of this research was to prove the applicability of the technique for evaluating an audiovisual environment, participants in the study were not local inhabitants nor stakeholders thus making them more objective in a way. In the actual co-creation process, inhabitants and other stakeholders may bring in their own knowledge about the place.

Participants were asked to rate the pleasantness of the experience of crossing the bridge on an II-point linear scale: "How would you rate your experience while passing this bridge to go from the city centre to the park?" The textual descriptions of the endpoints were "very unpleasant" $(-5)$ and "very pleasant" (+5). Fig. 6 shows that the green design D3_vegetated including some traffic noise reduction through a small noise barrier is clearly preferred.

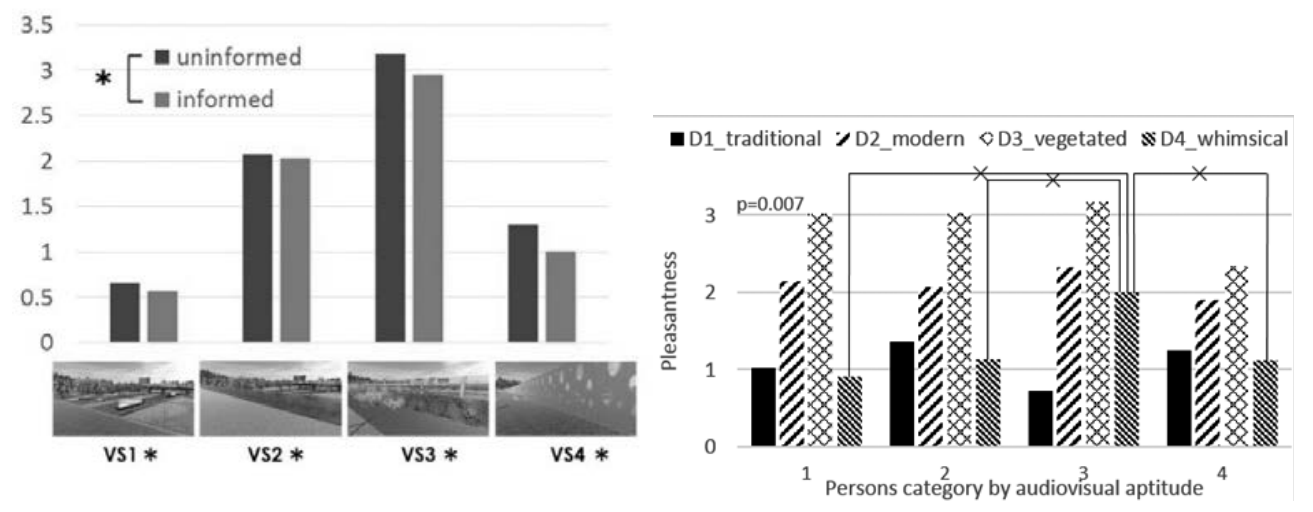

Fig. 6: Average evaluation of pleasantness rating (on a scale from very unpleasant $(-5)$ to very pleasant (5)) of walking across the bridge; LEFT: in the informed situation, participants were asked

about auditive and visual elements before asking them to experience the walk again; RIGHT: rating of the designs according to the audiovisual aptitude groups. Star indicates statistically significant differences. Source: Authors.

For this book chapter, some secondary effects that might need to be considered during a co-creation process relying on reproduction of a sonic environment in virtual reality are highlighted. In view of the importance of attention on the perception of soundscape one should be aware that presenting sound through headphones automatically focuses a person's attention on the sonic environment. In the case of the bridge, the aim is to background the sonic environment and thus to steer attention away from the possibly disturbing highway noise. To explore whether the VR environment creates an environment where sound could be backgrounded, the participants were asked to do similar experiments on several separate days. Although 
participants were not aware of this, on each day the visual environment was the same while different sonic environments were presented. The label "uninformed" in Fig. 6 refers to this situation where only designs with physically matching sonic environments are displayed. On the last day of the experiment, participants were asked to rate the importance of the different visual and sound elements in their overall assessment (e.g. the presence of green or the sound of the tram). This was expected to make them more aware of the sonic and visual environment and could make them evaluate the virtual walk across the bridge differently. The label "informed" in Fig. 6 refers to this situation. Although this leads to a statistically significant difference in rated pleasantness level on average over the 75 participants, the difference is negligible compared to the difference in rating between the different designs.

Similarly, it was investigated whether different people would rate the designs differently (Sun et al, 20l8b). Using a deviant detection experiment where sonic and visual elements were removed from a scene, participants were classified into: I) is very good in detecting auditory deviant stimuli but gets distracted by incongruent visual information; 2 ) is generally not good in detecting deviant sound; 3 ) is very apt in detecting deviant sound with or without the presence of visual information; 4) tends to make less mistakes when visual information is included even if it is incongruent. The right pane of Fig. 6 shows that only for people belonging to category 3 and only for the design 4 containing the high noise barrier, a significant difference is observed. Over all groups, the order of preference stays the same.

\section{AUGMENTING THE SOUND ENVIRONMENT OF THE PUBLIC SPACE BY CO-CREATION}

Augmenting the sound environment of the public space could also be achieved by adding electronically reproduced sound. With respect to the soundscape categorization explained above, this approach is mainly suitable for transforming backgrounded soundscapes to supportive soundscapes. Users of the public place could actively co-create the sonic environment by interacting with the playback device. This type of co-creation is far more hands on, interactive, and unstructured than other explained situations of co-creation.

Before deploying the playback equipment and the collection of sounds to the field, a lab test was performed. For this, the virtual environment discussed before is used. A recording at the locations where the playback device will be deployed is added. For visual presentation, an Oculus Rift was used. Sound reproduction used an open headphone (Sennheiser HD650). This allowed the person wearing this headphone to hear other sounds from the environment than those played back from the headphones. The test was conducted in a silent, semi-anechoic room using the playback device placed roughly at the same height and distance as it will be deployed in the field. 
Eight natural sound fragments including water sounds, wind, birds, and insects are made available for mixing with the existing sound environment during co-creating. These added sounds can be played simultaneously at a desired level by moving the mechanical sliders of the mixing panel. Soundscapes that were classified as backgrounded were selected. A snapshot from the visual environment for the four most relevant examples is shown in Fig. 7.

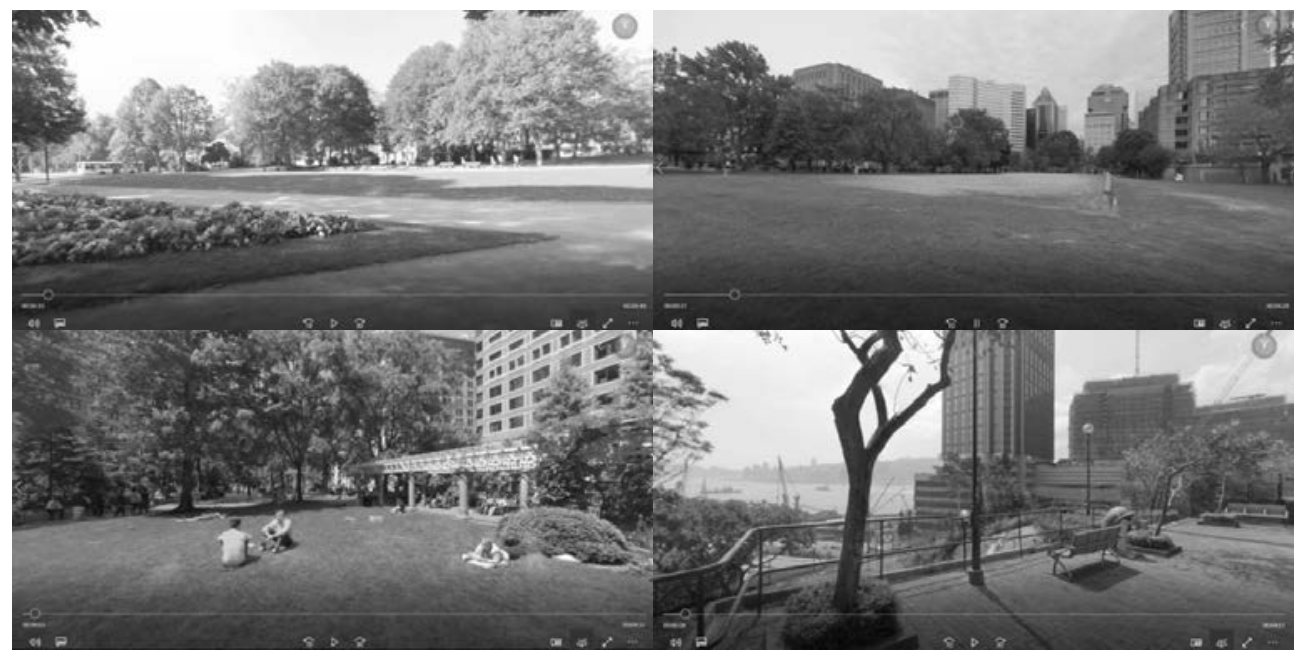

Fig. 7: Snapshots of the public places where the soundscape augmenting sounds are tested: upper left CP2: Ghent, Belgium; upper right R0008: Montreal, Canada; lower left: R00 17: Boston, USA; lower right: R0043: Hong Kong. Photos: Authors.

The compositions created by the 10 participants in the virtual co-creation is identified by the position of the sliders. The diversity of compositions is large both between people and between environments. Nevertheless, there are also some trends that can be observed from the averages shown in Table I. The sound of a single sparrow seems appreciated in all contexts. The mixture of songbirds scores high except in context R00 7 (Boston) where people are prominently present. Seagulls pop-up in R0043 (Hong Kong) where the waterfront context seems to create an expectation for such seabirds. Amongst the water sounds, the stream, a sound that is fluctuating in level, seems well appreciated overall, except in the very open park scene (R0008). This lab test showed which types of natural sounds should be made available at the co-creation loudspeaker systems. One important aspect of these sounds is that they should be noticeable in the hardly fluctuating but rather loud background sound that is often found in urban public places. The equivalent sound levels of the four VR samples (CP02, R0008, R0017, R0043) used in this experiment for example were $55.8 \mathrm{~dB}(\mathrm{~A}), 54.7 \mathrm{~dB}(\mathrm{~A}), 65.8 \mathrm{~dB}(\mathrm{~A}), 62.1 \mathrm{~dB}(\mathrm{~A})$ respectively. Bird song has a high fluctuation strength and thus adding these sounds to the constant background hum, is expected to make the soundscape more eventful. To some extent, the same effect is obtained by adding the water stream. The typical high frequency tonal compo- 
nents in the bird sound is also expected to make these sounds clearly noticeable and distinguishable. This allows the co-creators to move the overall soundscape from backgrounded to supportive.

Secondly - referring more to the pleasantness dimension of soundscape - sounds are recognized, they are given meaning. Although one might expect most natural sounds to contribute to the pleasantness of the soundscape, the sounds of raindrops, rustling leaves, and waterfall are not sufficiently different from the urban background to be recognized as natural sounds. Thus, they seem less appropriate candidates to be made available for co-creation. Plausibility of the sound and congruence with the visual scene also turn out to be very important. For example, the seagulls are only selected in R0043 where the visual context gives the impression of being close to the sea. But also the relatively low average level of the sound of the stream in R0008 could be an indication of lack of plausibility: the open view does not make the presence of water sound very plausible.

\begin{tabular}{|c|c|c|c|c|c|c|c|c|}
\hline & $\begin{array}{l}0 \\
\stackrel{0}{\Perp} \\
\stackrel{1}{\perp} \\
\end{array}$ & 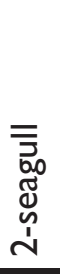 & $\begin{array}{l}3 \\
0 \\
\frac{1}{\pi} \\
\tilde{n} \\
0 \hat{n} \\
m\end{array}$ & 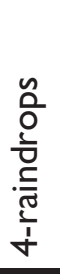 & 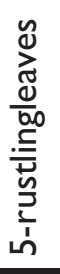 & 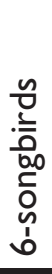 & 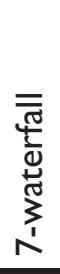 & 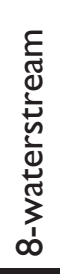 \\
\hline СР02 & 6 & -2 & 50 & 12 & 4 & 46 & 16 & 38 \\
\hline R0008 & -4 & -3 & 47 & 11 & 7 & 29 & 7 & $I$ \\
\hline R00I7 & -3 & 0 & 46 & 6 & -6 & 19 & 18 & 43 \\
\hline R0043 & -16 & 31 & 47 & I & 0 & 36 & 7 & 41 \\
\hline average & -4 & 6 & 48 & 7 & 1 & 32 & 12 & 31 \\
\hline
\end{tabular}

Table I: Averages over all participants of contributions of each added sound based on the reading of the amplification sliders (in slider-indicated dBs)

To bring co-creation to the field, some technical questions need to be addressed. A mechanical slider is not robust enough to be deployed in a park, but fortunately, many people carry a smart phone or tablet with wireless connectivity. Hence, an app ("Zuidpark soundscape app") is created that allows users via virtual sliders to control the sounds played at the nearest loudspeaker. The loudspeaker box contains a Raspberry Pi and an amplifier. All sounds are stored locally on Raspberry Pi which does not require internet connection. On the other hand, the device itself is configured as a WiFi access point to which the smartphone app can connect to. This implementation immediately determines the spatial extent from where the loudspeaker can be controlled: the spatial range of a WiFi access point.

To control the temporal aspect of the creation, again a technological solution is found. The sounds continue playing as long as the controlling device remains 
connected to the WiFi. To allow other users of the place to enjoy an interesting sound environment without creating it, there is also an option to start playing and rating previously composed mixtures. This soundscape co-creation platform is deployed in Gent, Belgium; more information can be found in Van Renterghem et al. (2020).

\section{DISCUSSION}

In this chapter, three examples were presented of co-creating the sonic environment of public places. Recording 360 degree visual scene with four channel ambisonics sound seems appropriate to create a high level of envelopment, immersion, representation, readability, realism, and overall quality. By classifying many recordings from public places in cities across the globe, an instructional database is obtained. The high-level classification in backgrounded, disruptive, calming and stimulating environments results in distinct classes for most of the available recordings. This shows that such a distinction is rather univocally made by different people. The more popular classification based on a soundscape interpretation of the two-dimensional circumplex model typically fails to find good examples for the monotonous quadrant. The classification could easily be extended using a taxonomy such as the one presented by Brown et al. (20II).

When VR is used to present alternative solutions to stakeholders in a co-creation process, care is needed to avoid excessive attention focus on a single component as such attention focus would not occur in the real situation. Using the example of a bridge design including noise barriers, it became clear that persons experiencing the environment did not focus on the sound: this would have led to rating the design with the lowest level of highway traffic noise as the most pleasant situation. On the contrary, the transparent noise barrier enriched with green elements was generally preferred. This finding is in accordance with previous research that showed that for transparent barriers perceived loudness and noise annoyance were judged lower than for opaque barriers (Maffei et al., 2013) and vegetation enhances the expected noise reduction and the aesthetic value of noise barriers (Hong \& Jeon, 2014).

This experiment also showed that presenting scenarios through VR is a stable technique. Pleasantness rating of the experience is generally lower after people have been asked in detail about various elements of the experience, amongst which the sound of the highway. Nevertheless, even after focusing their attention, people rank the designs in the same order. Similarly, personal factors seem to have an influence on how the virtual walk across the bridge is experienced, but this difference only changes the order of preference for one sub-group. Thus, although it may be advisable to include a variety of people in the co-creation process, it is expected that the outcome will not depend too strongly on the selection of participants.

Public places have been used for performing street-art since centuries. These activities significantly change the soundscape of the public space. The last example 
given in this chapter introduces digital technology to more subtly and in a new way changing the sonic environment in parks through a co-creation process. Hidden loudspeakers are used to add natural sounds to the environment. The collection of sounds that could be made available to the live co-creation process was validated in a pre-study in a controlled environment using virtual reality. This showed that the sound of birds is generally preferred but also that the species should match the expectation created by the (visual) environment. This finding is in agreement with earlier studies that explored the perceived restoration potential of bird sound (Ratcliffe et al., 2018) and the effect of bird biodiversity on well-being (Hedblom et al., 2017). The choice of water and wind sounds in the pre-study was also very specific. Findings suggest that the type of water sound is important: the sound of the water stream outperforms e.g. falling water (like a fountain) which is consistent with earlier findings (Galbrun \& Ali, 20I3).

All the sounds that are added during co-creation to backgrounded urban soundscapes have in common their ability to add Hi-Fi components (Dumyahn \& Pijanowski, 20II), to add salient components (Filipan et al., 20I9), and to increase natural variability of the overall soundscape (Botteldooren et al., 2006). But also, they all use pleasant and matching sounds, such that the soundscape becomes supportive for the overall experience of the public place. Further analysis of co-created soundscapes will show more precisely which acoustic components contribute most to the overall perception and appraisal of the urban soundscape.

Further analysis might be needed to elucidate the impact of the socio-economic profile of the people involved in the co-creation process, their demographics and familiarity with the places. Already when dealing with sounds alone, personal characteristics play a significant role (like e.g. the noise sensitivity construct, see Schreckenberg et al., 2010). This is further enhanced when audio-visual information is combined when outdoor environments are perceived, for which 4 groups of people can be identified (Sun et al., 2018b). Analysis including other personal factors, and their potential interactions with the presented findings here, would need a much larger number of participants.

\section{CONCLUSIONS}

The digital technologies discussed in this chapter are suitable tools for co-creating the sonic environment of public spaces. They allow to instruct stakeholders, present alternative scenarios during a planning phase, and even to augment the sonic experience in real time. In addition, they have the potential not only to improve the perception of environmental noise, but also the overall user experience and appreciation of a public place. 


\section{ACKNOWLEDGEMENTS}

The research leading to this chapter is partially funded by C3Places, which has received funding from the European Union's H2020 research innovation programme under grant agreement no. 693443. This study is supported by the HEAD Genuit Foundation through grant No. P-I6/II-W, "Urban Soundscapes of the World". The support of this foundation is gratefully acknowledged.

\section{REFERENCES}

Aletta, F., Kang, J., Astolfi, A., \& Fuda, S. (2016). Differences in soundscape appreciation of walking sounds from different footpath materials in urban parks. Sustainable Cities and Society, 27, 367-376.

Axelsson, Ö., Nilsson, M. E., \& Berglund, B. (2010). A principal components model of soundscape perception. The Journal of the Acoustical Society of America, 128(5), 2836-2846.

Björk, J., Albin, M., Grahn, P., Jacobsson, H., Ardö, J., Wadbro, J., Östergren, P.-O., \& Skärbäck, E. (2008). Recreational values of the natural environment in relation to neighbourhood satisfaction, physical activity, obesity and wellbeing. Journal of Epidemiology \& Community Health, 62, e2.

Botteldooren, D., De Coensel, B., \& De Muer, T. (2006). The temporal structure of urban soundscapes. Journal of sound and vibration, 292(I-2), 105-123.

Botteldooren, D., Dekoninck, L., Meeussen, C., Van Renterghem, T. (2018). Early stage sound planning in urban re-development: the Antwerp case study. Proceedings of the 47th international congress and exposition on noise control engineering (Internoise 2018), Chicago, US.

Bregman, A. S. (1994). Auditory scene analysis: The perceptual organization of sound. MIT press.

Brown, A. L., Kang, J., \& Gjestland, T. (20II). Towards standardization in soundscape preference assessment. Applied Acoustics, 72(6), 387-392.

Calabrese, C., \& Baresi, L. (2017). Outdoor augmented reality for urban design and simulation. In Urban Design and Representation (pp. I8I-190). Springer, Cham.

De Coensel, B., Vanwetswinkel, S., \& Botteldooren, D. (20I I). Effects of natural sounds on the perception of road traffic noise. The Journal of the Acoustical Society of America, I29(4), EL I48-ELI53.

De Coensel, B., Can, A., Degraeuwe, B., De Vlieger, I., \& Botteldooren, D. (20I2). Effects of traffic signal coordination on noise and air pollutant emissions. Environmental Modelling \& Software, 35, 74-83.

Dumyahn, S. L., \& Pijanowski, B. C. (20I I). Soundscape conservation. Landscape Ecology, 26(9), I327.

Filipan, K., Boes, M., De Coensel, B., Lavandier, C., Delaitre, P., Domitrović, H., \& Botteldooren, D. (2017). The personal viewpoint on the meaning of tranquility affects the appraisal of the urban park soundscape. Applied Sciences, 7(I), 91.

Filipan, K., De Coensel, B., Aumond, P., Can, A., Lavandier, C., \& Botteldooren, D. (2019). Auditory sensory saliency as a better predictor of change than sound amplitude in pleasantness assessment of reproduced urban soundscapes. Building and Environment, I48, 730-74I.

Fukuda, T., Nada, H., Adachi, H., Shimizu, S., Takei, C., Sato, Y., Yabuki, N., \& Motamedi, A. (20I7). Integration of a Structure from Motion into Virtual and Augmented Reality for Architectural and Urban Simulation. In International Conference on Computer-Aided Architectural Design Futures (pp. 60-77). Springer, Singapore.

Galbrun, L., \& Ali, T. T. (20I3). Acoustical and perceptual assessment of water sounds and their use over road traffic noise. The Journal of the Acoustical Society of America, I33(I), 227-237.

Georgiou, F., \& Hornikx, M. (2017). Auralization of a car pass-by using impulse responses computed with the pseudospectral time-domain method. The Journal of the Acoustical Society of America, I4I(5), 3778-3779. 
Hedblom, M., Knez, I., \& Gunnarsson, B. (2017). Bird diversity improves the well-being of city residents. In Ecology and conservation of birds in urban environments (pp. 287-306). Springer, Cham.

Hong, J. Y., \& Jeon, J. Y. (20I4). The effects of audio-visual factors on perceptions of environmental noise barrier performance. Landscape and Urban Planning, I25, 28-37.

Kang, J., Aletta, F., Gjestland, T., Brown, L., Botteldooren, D., Schulte-Fortkamp, B., Lercher, P., van Kamp, I., Genuit, K., Fiebig, A., Bento Coelho, J., Maffei, L., Lavia, L. (2016). Ten questions on the soundscapes of the built environment. Building and Environment, 108, 284-294.

Licitra, G., Cobianchi, M., \& Brusci, L. (2010, June). Artificial soundscape approach to noise pollution in urban areas. In INTER-NOISE and NOISE-CON Congress and Conference Proceedings (pp. 60806089).

Maffei, L., Masullo, M., Aletta, F., \& Di Gabriele, M. (2013). The influence of visual characteristics of barriers on railway noise perception. Science of the Total Environment, 445, 4I-47.

Raimbault, M., \& Dubois, D. (2005). Urban soundscapes: Experiences and knowledge. Cities, 22(5), 339350.

Ratcliffe, E., Gatersleben, B., \& Sowden, P. T. (20/8). Predicting the perceived restorative potential of bird sounds through acoustics and aesthetics. Environment and Behavior, 00I39I65I8806952.

Rehan, R. M. (2016). The phonic identity of the city urban soundscape for sustainable spaces. Hbrc Journal, I2(3), 337-349.

Sanchez, G. M. E., Alves, S., \& Botteldooren, D. (2018). Urban Sound Planning: An Essential Component in Urbanism and Landscape Architecture. In Handbook of Research on Perception-Driven Approaches to Urban Assessment and Design (pp. I-22). IGI Global.

Sanchez, G. M. E., Van Renterghem, T., Sun, K., De Coensel, B., \& Botteldooren, D. (2017). Using Virtual Reality for assessing the role of noise in the audio-visual design of an urban public space. Landscape and Urban Planning, 167, 98-107.

Schreckenberg, D., Griefahn, B., Meis, M. (2010). The associations between noise sensitivity, reported physical and mental health, perceived environmental quality, and noise annoyance. Noise Health 12, 7-16. Schulte-Fortkamp, B., \& Jordan, P. (2016). When soundscape meets architecture. Noise Mapping, 3(I). Steele, D., Bild, E., Tarlao, C., \& Guastavino, C. (2019). Soundtracking the public space: Outcomes of the Musikiosk soundscape intervention. International Journal of Environmental Research and Public Health, 16, 1865.

Sun, K., Botteldooren, D., De Coensel, B. (2018a). Realism and immersion in the reproduction of audio-visual recordings for urban soundscape evaluation. In 47th International Congress and Exposition on Noise Control Engineering (Inter-Noise 2018) (Pp. I-10).

Sun, K., Echevarria Sanchez, G. M., De Coensel, B., Van Renterghem, T., Talsma, D., \& Botteldooren, D. (20I8b). Personal audiovisual aptitude influences the interaction between landscape and soundscape appraisal. Frontiers in psychology, 9, 780.

Sun, K., De Coensel, B., Filipan, K., Aletta, F., Van Renterghem, T., De Pessemier, T., Joseph, W., Botteldooren, D. (2019). Classification of soundscapes of urban public open spaces. Landscape and urban planning, 189, I39-I55.

Van Renterghem, T., \& Botteldooren, D. (2012). On the choice between walls and berms for road traffic noise shielding including wind effects. Landscape and Urban Planning, 105(3), 199-210.

Van Renterghem, T., Vanhecke, K., Filipan, K., Sun, K., De Pessemier, T., De Coensel, B., Joseph, W., Botteldooren, D. (2020). Interactive soundscape augmentation by natural sounds in a noise polluted urban park. Landscape and urban planning, 194, 103705. 